This item was submitted to Loughborough's Research Repository by the author.

Items in Figshare are protected by copyright, with all rights reserved, unless otherwise indicated.

An introduction to inhomogeneous liquids, density functional theory, and the wetting transition

PLEASE CITE THE PUBLISHED VERSION

http://dx.doi.org/10.1119/1.4890823

PUBLISHER

(c) American Association of Physics Teachers

VERSION

VoR (Version of Record)

LICENCE

CC BY-NC-ND 4.0

REPOSITORY RECORD

Hughes, Adam P., Andrew J. Archer, and Uwe Thiele. 2019. "An Introduction to Inhomogeneous Liquids, Density Functional Theory, and the Wetting Transition”. figshare. https://hdl.handle.net/2134/16887. 


\section{AAPT AMERICAN \\ of PHYSICS}

\section{An introduction to inhomogeneous liquids, density functional theory, and the wetting transition}

Adam P. Hughes, Uwe Thiele, and Andrew J. Archer

Citation: American Journal of Physics 82, 1119 (2014); doi: 10.1119/1.4890823

View online: http://dx.doi.org/10.1119/1.4890823

View Table of Contents: http://scitation.aip.org/content/aapt/journal/ajp/82/12?ver=pdfcov

Published by the American Association of Physics Teachers

\section{Articles you may be interested in}

Density functional theory of inhomogeneous liquids. II. A fundamental measure approach

J. Chem. Phys. 128, 184711 (2008); 10.1063/1.2916694

Mean-field density-functional model of a second-order wetting transition

J. Chem. Phys. 128, 114716 (2008); 10.1063/1.2895748

Surface phase transitions in athermal mixtures of hard rods and excluded volume polymers investigated using a density functional approach

J. Chem. Phys. 125, 204709 (2006); 10.1063/1.2400033

Homogeneous nucleation at high supersaturation and heterogeneous nucleation on microscopic wettable particles: A hybrid thermodynamic/density-functional theory

J. Chem. Phys. 125, 144515 (2006); 10.1063/1.2357937

Perfect wetting along a three-phase line: Theory and molecular dynamics simulations

J. Chem. Phys. 124, 244505 (2006); 10.1063/1.2206772

\section{WebAssign.}

Free Physics Videos

Add these videos and many more

resources - free with WebAssign.

bit.do/PhysicsResources

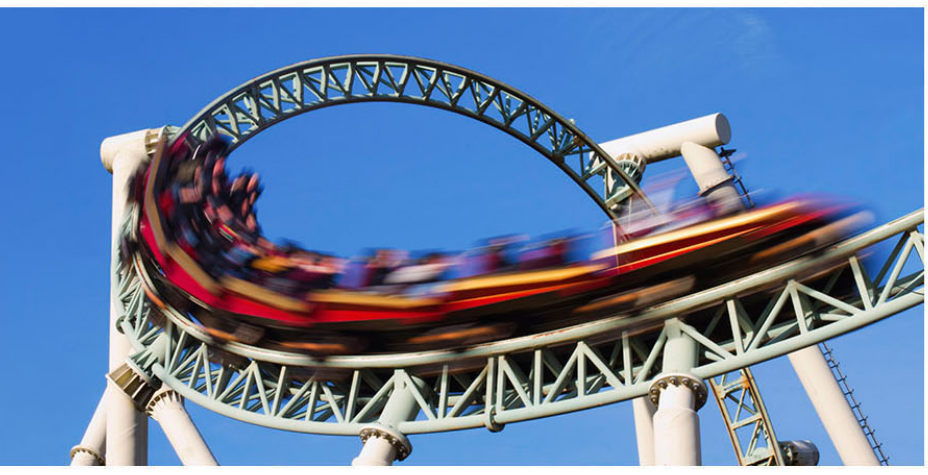




\title{
An introduction to inhomogeneous liquids, density functional theory, and the wetting transition
}

\author{
Adam P. Hughes \\ Department of Mathematical Sciences, Loughborough University, Loughborough, Leicestershire LE11 3TU, \\ United Kingdom \\ Uwe Thiele \\ Department of Mathematical Sciences, Loughborough University, Loughborough, Leicestershire LE11 3TU, \\ United Kingdom and Institut für Theoretische Physik, Westfälische Wilhelms-Universität Münster, \\ Wilhelm Klemm Str. 9, D-48149 Münster, Germany
}

\begin{abstract}
Andrew J. Archer
Department of Mathematical Sciences, Loughborough University, Loughborough, Leicestershire LE11 3TU, United Kingdom
\end{abstract}

(Received 8 August 2013; accepted 9 July 2014)

\begin{abstract}
Classical density functional theory (DFT) is a statistical mechanical theory for calculating the density profiles of the molecules in a liquid. It is widely used, for example, to study the density distribution of the molecules near a confining wall, the interfacial tension, wetting behavior, and many other properties of nonuniform liquids. DFT can, however, be somewhat daunting to students entering the field because of the many connections to other areas of liquid-state science that are required and used to develop the theories. Here, we give an introduction to some of the key ideas, based on a lattice-gas (Ising) model fluid. This approach builds on knowledge covered in most undergraduate statistical mechanics and thermodynamics courses, so students can quickly get to the stage of calculating density profiles, etc., for themselves. We derive a simple DFT for the lattice gas and present some typical results that can readily be calculated using the theory. ( 2014 American Association of Physics Teachers.

[http://dx.doi.org/10.1119/1.4890823]
\end{abstract}

\section{INTRODUCTION}

The behavior of liquids at interfaces and in confinement is a fascinating and important area of study. For example, the behavior of a liquid under confinement between two surfaces determines how good a lubricant that liquid is. The nature of the interactions between the liquid and the surfaces is crucial. Consider, for example, the Teflon coating on non-stick cooking pans, used because water does not adhere to (wet) the surface. One can approach this problem from a mesoscopic fluid-mechanical point of view; see, for example, the excellent book by de Gennes, Brochard-Wyart, and Queré. ${ }^{1}$ However, if a microscopic approach is required, which relates the fluid properties at an interface to the nature of the molecular interactions, then one must start from statistical mechanics. There are a number of books ${ }^{2-5}$ that provide a good starting point. All of these include a discussion of classical density functional theory (DFT), which is a theory for determining the density profile of a fluid in the presence of an external potential, such as that exerted by the walls of a container.

DFT is a statistical mechanical theory, where the aim is to calculate average properties of the system being studied. In statistical mechanics, the central quantity of interest is the partition function $Z$; once this is calculated, all thermodynamic quantities are easily found. However, because $Z$ is a sum over all the possible configurations of the system, it can rarely be evaluated exactly. Instead of focusing on $Z$, in DFT we seek to develop good approximations for the free energy. It can be shown that the free energy is a functional of the fluid density profile $\rho(\mathbf{r})$, and the equilibrium profile is the one that minimizes the free energy. Over the years, many different approximations for the free energy functionals have been developed, generally by making contact with results from other branches of liquid-state physics. There are now quite a few lecture notes and review articles on the subject. $^{6-12}$ This rather large literature can make learning about DFT rather daunting.

One of us (A.J.A.) has found in teaching this subject that a good place for students to start learning about the properties of inhomogeneous fluids is by considering a simple lattice gas (Ising) model. This allows students to avoid much of the liquid-state physics and functional calculus that can be daunting for undergraduates. ${ }^{13}$ The advantage of starting from a lattice-gas model is that one can quickly develop a simple mean-field DFT (described below) and then proceed to calculate the bulk fluid phase diagram and study the interfacial properties of the model, such as determining the wetting behavior and finding wetting transitions. The computer algorithms required to find solutions to the lattice gas model are also fairly simple. Thus, the threshold for entering the subject and getting to the point where students can calculate things for themselves is much lower via this route, compared to the other routes that we can think of.

The aim of this paper is two-fold. First, we derive the mean-field DFT for an inhomogeneous lattice-gas fluid while explaining the physics of the theory. This presentation assumes that the reader has had introductory statistical mechanics and thermodynamics courses, but little else beyond that. Second, we illustrate the types of quantities that DFT can be used to calculate, such as the surface tension of the liquid-gas interface. We also show how the model is used to study wetting behavior and to answer questions like "what is the shape of a drop of liquid on a surface?" The paper also includes four exercises for students.

This paper is laid out as follows. In Sec. II, we introduce the statistical mechanics of simple liquids and then set up the DFT model in Secs. III and IV. The bulk fluid phase diagram is discussed in Sec. V. We describe the iterative method for solving the model in Sec. VI and then display some typical 
results in Sec. VII. Finally, some conclusions are drawn in Sec. VIII.

\section{STATISTICAL MECHANICS OF SIMPLE LIQUIDS}

We consider a classical fluid composed of $N$ atoms or molecules in a container. What follows is also relevant to colloidal suspensions, so we simply refer to the atoms, molecules, colloids, etc., as "particles." The fluid's energy is a function of the set of position and momentum coordinates, $\mathbf{r}^{N} \equiv$ $\left\{\mathbf{r}_{1}, \mathbf{r}_{2}, \ldots, \mathbf{r}_{N}\right\}$ and $\mathbf{p}^{N} \equiv\left\{\mathbf{p}_{1}, \mathbf{p}_{2}, \ldots, \mathbf{p}_{N}\right\}$, respectively, and is given by the Hamiltonian

$$
\mathcal{H}\left(\mathbf{r}^{N}, \mathbf{p}^{N}\right)=K\left(\mathbf{p}^{N}\right)+E\left(\mathbf{r}^{N}\right),
$$

where

$$
K=\sum_{i=1}^{N} \frac{\mathbf{p}_{i}^{2}}{2 m}
$$

is the kinetic energy and $E$ is the potential energy due to the interactions between the particles and also to any external potentials such as those due to the container walls. When treating the system in the canonical ensemble, which has fixed volume $V$, particle number $N$, and temperature $T$, the probability that the system is in a particular state is ${ }^{4,5,14,15}$

$$
f\left(\mathbf{r}^{N}, \mathbf{p}^{N}\right)=\frac{1}{h^{3 N} N !} \frac{e^{-\beta \mathcal{H}}}{Z},
$$

where

$$
Z=\frac{1}{h^{3 N} N !} \int d \mathbf{r}^{N} \int d \mathbf{p}^{N} e^{-\beta \mathcal{H}}
$$

is the canonical partition function, $h$ is Plank's constant, and $\beta=\left(k_{B} T\right)^{-1}$ with $k_{B}$ Boltzmann's constant. The partition function allows macroscopic thermodynamic quantities to be related to the microscopic properties of the system that are defined in $\mathcal{H}$.

The kinetic energy contribution (2) is solely a function of the momenta $\mathbf{p}^{N}$, while $E\left(\mathbf{r}^{N}\right)$, the precise form of which is yet to be defined, depends only on the positions of the particles $\mathbf{r}^{N}$. This dependence allows the partition function (4) to be simplified by performing the Gaussian integrals over the momenta to obtain

$$
\begin{aligned}
Z & =\frac{1}{h^{3 N}} \int d \mathbf{p}^{N} \exp \left(-\beta \sum_{i=1}^{N} \frac{\mathbf{p}_{i}^{2}}{2 m}\right) Q \\
& =\frac{1}{h^{3 N}} \int e^{-\beta \mathbf{p}_{1}^{2} / 2 m} d \mathbf{p}_{1} \cdots \int e^{-\beta \mathbf{p}_{N}^{2} / 2 m} d \mathbf{p}_{N} Q \\
& =\frac{1}{h^{3 N}}\left(\sqrt{\frac{2 m \pi}{\beta}}\right)^{3} \cdots\left(\sqrt{\frac{2 m \pi}{\beta}}\right)^{3} Q \\
& =\left(\sqrt{\frac{2 m \pi}{\beta h^{2}}}\right)^{3 N} Q=\Lambda^{-3 N} Q,
\end{aligned}
$$

where $\Lambda=\sqrt{\beta h^{2} / 2 m \pi}$ is the thermal de Broglie wavelength and

$$
Q=\frac{1}{N !} \int d \mathbf{r}^{N} e^{-\beta E}
$$

is the configuration integral. ${ }^{14}$ Thus, the partition function is just the configuration integral multiplied by a factor $\left(\Lambda^{-3 N}\right)$ that depends on $N, T$, and $m$. Changing the value of the length $\Lambda$ simply results in a uniform shift in the free energy per particle [see Eq. (10)] and so does not determine the phase behavior of the system, which is given by free energy differences between states. Nor does the value of $\Lambda$ determine the fluid structure, which is encoded in $Q$. We can therefore set $\Lambda=1$ without any change in the results.

Evaluating $Q$ is the central problem here and, in general, this cannot be done exactly so approximations are required. In Sec. III, we develop a simple lattice model approximation that allows progress.

\section{DISCRETE MODEL}

\section{A. Defining a lattice}

To simplify the analysis, we assume that the fluid is two dimensional (2D), although everything can easily be extended to a three-dimensional (3D) system. We imagine a lattice that discretizes space, so any configuration of particles can be described by a set of lattice occupation numbers $\left\{n_{1}, n_{2}, \ldots, n_{N}\right\} \equiv\left\{n_{i}\right\}$, which specify whether the lattice sites are filled $\left(n_{i}=1\right)$ or empty $\left(n_{i}=0\right)$ as illustrated in Fig. 1. There are $M$ lattice sites and the width of each site is denoted by $\sigma$, which is also equal to the diameter of a particle. We use units in which $\sigma=1$. The particles are assumed to be spherical, so that their orientation is not important. In our discretized space, the integrals in Eq. (6) become sums over all possible states of the lattice. Note the short hand $i \equiv(k, l)$, where $k$ and $l$ are integer indices defining the 2D lattice.

\section{B. Energy of the system}

To proceed, we must specify the nature of the potential energy function $E$. We assume the form

$$
E=\sum_{i=1}^{M} n_{i} V_{i}-\frac{1}{2} \sum_{i, j} \varepsilon_{i j} n_{i} n_{j},
$$

where the first term is the contribution from an external potential $V_{i}$ and the second term is the contribution from

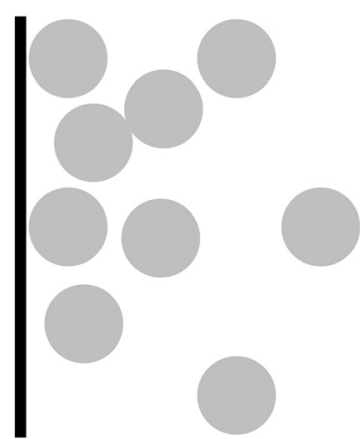

(a) Continuum fluid configuration

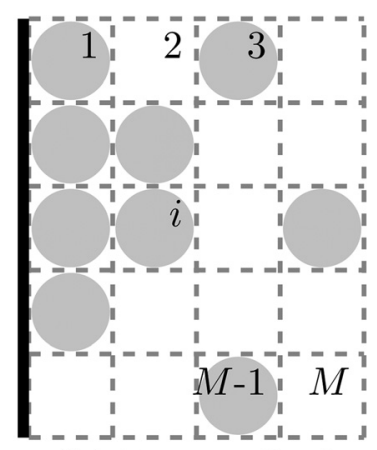

(b) Lattice fluid configuration
Fig. 1. Illustration of how a continuous system (a) can be discretized in space by setting the particles on a lattice (b). 
interactions between pairs of particles. We assume that there are no three-body or other such higher-body interactions. The interaction energy between particles at two lattice sites $i$ and $j$ is $\varepsilon_{i j}$. This value gets smaller as the distance between sites increases and approaches zero as the distance goes to infinity. The term $-(1 / 2) \sum_{i, j} \varepsilon_{i j} n_{i} n_{j}$ denotes a sum over all pairs of lattice sites in the system; the factor of $1 / 2$ is present to avoid double counting. Allowing only pair interactions greatly simplifies the task of evaluating the partition function, but it can still be arduous to evaluate this sum even for a moderately sized system. The probability of being in a particular configuration, $\left\{n_{i}\right\}$, for a fixed number of particles $N$, is now

$$
P\left(\left\{n_{i}\right\}\right)=\frac{e^{-\beta E\left(\left\{n_{i}\right\}\right)}}{Z},
$$

with the partition function defined as

$$
Z=\sum_{\text {all states }} e^{-\beta E_{\text {state }}},
$$

where "state" is a shorthand for a particular allowed set of occupation numbers $\left\{n_{i}\right\}$. Note the relation to the configuration integral in Eq. (6), with the sum over all states approximating the continuum integral $(N !)^{-1} \int d \mathbf{r}^{N}(\cdots)$.

\section{Helmholtz free energy}

The Helmholtz free energy can be calculated from the partition function as $5,14,15$

$$
F=-k_{B} T \ln Z \text {. }
$$

All other thermodynamic quantities can then be obtained from derivatives of $F$. However, we are still unable to evaluate the sum in Eq. (9), and as a consequence we cannot calculate $F$.

Still, under certain assumptions, we can make some progress. Consider the special case where there is no external field, i.e., $V_{i}=0$, and with $\varepsilon_{i j}=0$ so the particles do not interact with each other. From Eq. (7), this gives $E=0$ for all configurations and from Eq. (8), we observe that

$$
P\left(\left\{n_{i}\right\}\right)=\frac{1}{Z},
$$

so that all configurations are equally likely. From Eq. (9), we see that $Z$ is just the number of possible states, which, for a system of $M$ lattice sites containing $N$ particles, is

$$
Z=\frac{M !}{N !(M-N) !}
$$

For large systems, when both $M$ and $N$ are large, this can be simplified using Stirling's approximation $\ln (N !) \approx$ $N \ln N-N$, which, with Eq. (10), gives

$$
F=-k_{B} T[M \ln M-N \ln N-(M-N) \ln (M-N)] .
$$

The number density of particles in the system is $\rho=N / M$ (recall $\sigma=1$ ), and so Eq. (13) leads to

$$
F=M k_{B} T[\rho \ln \rho+(1-\rho) \ln (1-\rho)] .
$$

This homogeneous fluid has a uniform density $\rho$ throughout. However, for a fluid in the presence of a spatially varying external potential $V_{i}$ we should expect the density to vary in space. The average density at lattice point $i$ is defined as

$$
\rho_{i}=\left\langle n_{i}\right\rangle
$$

i.e., it is the average value of the occupation number at site $i$ over all possible configurations: $\langle\cdots\rangle=\sum_{\text {all states }}(\cdots) P_{\text {state }}$. Next, we obtain an approximation for the free energy of the inhomogeneous fluid.

\section{The grand canonical ensemble}

So far we have treated the system in the canonical ensemble, with a fixed $N, T$, and volume $V$. We now switch to the grand canonical ensemble, with fixed $V$ and $T$ but varying $N$ as the system exchanges particles with a reservoir, whose chemical potential is $\mu$. Physically, one can consider the "system" to be a subsystem of a much larger structure that includes the reservoir, with $T$ and $\mu$ being practically fixed because the reservoir is so large.

The probability of a grand canonical system being in a particular state is [cf. Eq. (8)]

$$
P\left(\left\{n_{i}\right\}\right)=\frac{e^{-\beta(E-\mu N)}}{\Xi},
$$

where the number of particles in the system (still with $M$ lattice sites) is

$$
N=\sum_{i=1}^{M} n_{i}
$$

The normalization factor $\Xi$ is the grand canonical partition function

$$
\Xi=\operatorname{Tr} e^{-\beta(E-\mu N)}
$$

where the trace operator $\operatorname{Tr}$ is defined as

$$
\operatorname{Tr} x=\sum_{\text {all states }} x=\sum_{n_{1}=0}^{1} \sum_{n_{2}=0}^{1} \ldots \sum_{n_{M}=0}^{1} x .
$$

From the grand canonical partition function, we can find the grand potential

$$
\Omega=-k_{B} T \ln \Xi
$$

in analogy to Eq. (10). The equilibrium state corresponds to the minimum of the grand potential.

\section{E. Gibbs-Bogoliubov inequality}

Our goal is to obtain an approximation for the grand potential $\Omega$. To do this, we first derive and then use the Gibbs-Bogoliubov inequality, which provides an upper bound on the true grand potential. We then minimize our expression for the upper bound to obtain a best estimate for the grand potential. 
We start by rearranging Eq. (20) and then equating to Eq. (18) to give

$$
e^{-\beta \Omega}=\operatorname{Tr} e^{-\beta(E-\mu N)} .
$$

The energy $E$ of a particular state can be rewritten as

$$
E=E_{0}+E-E_{0}=E_{0}+\Delta E,
$$

where $E_{0}$ is the energy of a reference system. The reference system is selected so as to be able to evaluate its partition function. Thus, the reference system has the same possible states $\left\{n_{i}\right\}$, but the interaction energy $E_{0}\left(\left\{n_{i}\right\}\right)$ is simple enough that we can evaluate $\Xi_{0}$. From Eqs. (21) and (22), we obtain

$$
e^{-\beta \Omega}=\operatorname{Tr} e^{-\beta\left(E_{0}-\mu N\right)} e^{-\beta \Delta E} .
$$

We now use the idea of the statistical average to find the Gibbs-Bogoliubov inequality. The statistical average value of any quantity $x$ in the reference system is

$$
\langle x\rangle_{0}=\operatorname{Tr}\left(\frac{e^{-\beta\left(E_{0}-\mu N\right)}}{\Xi_{0}} x\right),
$$

since the probability of finding the reference system in a particular state with energy $E_{0}$ is $P_{0}=e^{-\beta\left(E_{0}-\mu N\right)} / \Xi_{0}$ [see Eq. (16)]. So, from Eq. (23) we obtain

$$
e^{-\beta \Omega}=e^{-\beta \Omega_{0}}\left\langle e^{-\beta \Delta E}\right\rangle_{0},
$$

with $\Xi_{0}=e^{-\beta \Omega_{0}}$ given by Eqs. (18) and (20). However, since $e^{-x}$ is a convex function of $x$, it follows that $\left\langle e^{-x}\right\rangle \geq e^{-\langle x\rangle}$, so from Eq. (25) we obtain the inequality

$$
e^{-\beta \Omega} \geq e^{-\beta \Omega_{0}} e^{-\beta\langle\Delta E\rangle_{0}}
$$

Taking the logarithm of both sides gives the GibbsBogoliubov inequality ${ }^{5}$

$$
\Omega \leq \Omega_{0}+\langle\Delta E\rangle_{0} .
$$

This inequality gives an upper bound on the true grand potential $\Omega$ that depends solely on the properties of the reference system, and, more importantly, it allows us to find a "best" approximation for $\Omega$ by minimizing the right-hand side of the inequality. We choose $E_{0}$ to depend on a set of parameters $\left\{\phi_{i}\right\}$ that can be varied and perform the minimization with respect to these parameters.

To proceed, we must specify $E_{0}$ so that $\Omega_{0}$ and $\langle\Delta E\rangle_{0}$ can be found. We choose

$$
E_{0}=\sum_{i=1}^{M}\left(V_{i}+\phi_{i}\right) n_{i}
$$

where $V_{i}$ is the external potential and $\phi_{i}$ are the parameters mentioned above, which are yet to be determined. Physically, $\phi_{i}$ are the (mean field) additional effective potentials that incorporate the effect of the interactions between the particles.

We now show how $\Omega_{0}$ and $\langle\Delta E\rangle_{0}$ can be expressed as a function of the fluid density profile $\left\{\rho_{i}\right\}$. We also replace $\left\{\phi_{i}\right\}$ by expressions involving $\left\{\rho_{i}\right\}$. The average density $\rho_{i}$ at a particular lattice site $i$ is given by Eq. (15). Our (mean field) approximation for this quantity is

$$
\begin{aligned}
\rho_{i} & =\left\langle n_{i}\right\rangle_{0}=\operatorname{Tr}\left(\frac{e^{-\beta E_{0}-\mu N}}{\Xi_{0}} n_{i}\right)=\frac{1}{\Xi_{0}}\left[\sum_{n_{1}=0}^{1} e^{-\beta\left(V_{1}+\phi_{1}-\mu\right) n_{1}}\right] \cdots\left[\sum_{n_{i}=0}^{1} n_{i} e^{-\beta\left(V_{i}+\phi_{i}-\mu\right) n_{i}}\right] \ldots\left[\sum_{n_{M}=0}^{1} e^{-\beta\left(V_{M}+\phi_{M}-\mu\right) n_{M}}\right] \\
& =\left[\frac{\sum_{n_{1}=0}^{1} e^{-\beta\left(V_{1}+\phi_{1}-\mu\right) n_{1}}}{\sum_{n_{1}=0}^{1} e^{-\beta\left(V_{1}+\phi_{1}-\mu\right) n_{1}}}\right] \cdots\left[\frac{\sum_{n_{i}=0}^{1} n_{i} e^{-\beta\left(V_{i}+\phi_{i}-\mu\right) n_{i}}}{\sum_{n_{i}=0}^{1} e^{-\beta\left(V_{i}+\phi_{i}-\mu\right) n_{i}}}\right] \cdots\left[\frac{\left.\sum_{n_{M}=0}^{1} e^{-\beta\left(V_{M}+\phi_{M}-\mu\right) n_{M}}\right]}{\sum_{n_{M}=0}^{1} e^{-\beta\left(V_{M}+\phi_{M}-\mu\right) n_{M}}}\right]=\frac{\sum_{n_{i}=0}^{1} n_{i} e^{-\beta\left(V_{i}+\phi_{i}-\mu\right) n_{i}}}{\sum_{n_{i}=0}^{1} e^{-\beta\left(V_{i}+\phi_{i}-\mu\right) n_{i}}}=\frac{e^{-\beta\left(V_{i}+\phi_{i}-\mu\right)}}{1+e^{-\beta\left(V_{i}+\phi_{i}-\mu\right)}} .
\end{aligned}
$$

Also, the reference system grand partition function is [cf. Eq. (18)]

$$
\begin{aligned}
\Xi_{0} & =\operatorname{Tr} e^{-\beta\left(E_{0}-\mu N\right)}=\operatorname{Tr} e^{-\beta \sum_{i=1}^{M}\left(V_{i}+\phi_{i}-\mu\right) n_{i}} \\
& =\prod_{i=1}^{M}\left[1+e^{-\beta\left(V_{i}+\phi_{i}-\mu\right)}\right] .
\end{aligned}
$$

This expression can now be substituted into Eq. (20) to obtain the following expression for the grand potential:

$$
\Omega_{0}=-k_{B} T \sum_{i=1}^{M} \ln \left[1+e^{-\beta\left(V_{i}+\phi_{i}-\mu\right)}\right] .
$$

Rearranging Eq. (29) to give $1-\rho_{i}=\left[1+e^{-\beta\left(V_{i}+\phi_{i}-\mu\right)}\right]^{-1}$ and inserting it into Eq. (31) gives

$$
\Omega_{0}=k_{B} T \sum_{i=1}^{M} \ln \left(1-\rho_{i}\right) .
$$

By rewriting this as 


$$
\Omega_{0}=k_{B} T \sum_{i=1}^{M}\left(\rho_{i}+1-\rho_{i}\right) \ln \left(1-\rho_{i}\right),
$$

we can use Eq. (29) to express $\Omega_{0}$ in the form

$$
\begin{aligned}
\Omega_{0}= & k_{B} T \sum_{i=1}^{M}\left[\rho_{i} \ln \rho_{i}+\left(1-\rho_{i}\right) \ln \left(1-\rho_{i}\right)\right] \\
& +\sum_{i=1}^{M}\left(V_{i}+\phi_{i}-\mu\right) \rho_{i} .
\end{aligned}
$$

Note that when $V_{i}=\phi_{i}=0$, which corresponds to the case of a uniform fluid with $\varepsilon_{i j}=0$, this expression reduces to the result we saw earlier in Eq. (14), since $\Omega=F-\mu N .^{5,20}$ Returning to the general case $\varepsilon_{i j} \neq 0$, and using the definition of $E_{0}$ in Eq. (28), we find that $\Delta E=E-E_{0}$ is

$$
\Delta E=-\frac{1}{2} \sum_{i, j} \varepsilon_{i j} n_{i} n_{j}-\sum_{i=1}^{M} \phi_{i} n_{i} .
$$

Taking the statistical average of this expression and using the definition of particle density $\rho_{i}=\left\langle n_{i}\right\rangle_{0}$, we find

$$
\langle\Delta E\rangle_{0}=-\frac{1}{2} \sum_{i, j} \varepsilon_{i j} \rho_{i} \rho_{j}-\sum_{i=1}^{M} \phi_{i} \rho_{i},
$$

where, because our reference system is non-interacting, leads to

$$
\left\langle n_{i} n_{j}\right\rangle_{0}=\left\langle n_{i}\right\rangle_{0}\left\langle n_{j}\right\rangle_{0}=\rho_{i} \rho_{j} .
$$

Finally, Eqs. (34) and (36) can be used to obtain the upper bound of the true grand potential: ${ }^{16}$

$$
\begin{aligned}
\Omega \leq \hat{\Omega}= & \Omega_{0}+\langle\Delta E\rangle_{0} \\
= & k_{B} T \sum_{i=1}^{M}\left[\rho_{i} \ln \rho_{i}+\left(1-\rho_{i}\right) \ln \left(1-\rho_{i}\right)\right] \\
& -\frac{1}{2} \sum_{i, j} \varepsilon_{i j} \rho_{i} \rho_{j}+\sum_{i=1}^{M}\left(V_{i}-\mu\right) \rho_{i} .
\end{aligned}
$$

One should choose the mean field $\left\{\phi_{i}\right\}$ so as to minimize the upper bound for the grand potential $\hat{\Omega}$ in order to generate a best approximation for $\Omega$. This process is equivalent to choosing the set $\left\{\rho_{i}\right\}$ so as to minimize $\hat{\Omega}$, since the density $\rho_{i}$ is defined by $\phi_{i}$ [cf. Eq. (29)]. What we have done here is to derive an approximate DFT for the lattice fluid. For DFT in general, one can prove that the equilibrium fluid density profile is that which minimizes the grand potential functional. ${ }^{6}$ Thus, that the equilibrium fluid density profile $\left\{\rho_{i}\right\}$ is that which minimizes $\hat{\Omega}$ is in fact a particular example (on the lattice) of a much more general principle.

\section{DEFINING THE POTENTIALS}

Up to this point, we have not specified the forms of the potentials from the external field or the particle interactions. We now define $\varepsilon_{i j}$ and $V_{i}$.

\section{A. Particle interactions}

The term $-(1 / 2) \sum_{i, j} \varepsilon_{i j} \rho_{i} \rho_{j}$ in Eq. (38) represents the contribution to the free energy from the interactions between pairs of particles. A simple example of the continuum fluid we seek to model is made up of particles interacting via a Lennard-Jones pair potential ${ }^{5}$ of the form $v(r)=\varepsilon\left[\left(r_{0} / r\right)^{12}\right.$ $\left.-2\left(r_{0} / r\right)^{6}\right]$, where $r$ is the distance between a pair of particles and $r_{0}$ is the distance at the minimum where $v\left(r_{0}\right)=-\varepsilon$. Given that $v\left(2 r_{0}\right) \approx-0.03 \varepsilon$, it is a good approximation to assume that each particle interacts only with the nearest and next-nearest neighboring particles, as illustrated in Fig. 2. We replace the particle interaction term in the free energy with

$$
\sum_{i, j} \varepsilon_{i j} \rho_{i} \rho_{j} \approx \varepsilon_{n n} \sum_{i=1}^{M} \rho_{i} \sum_{j n n i} \rho_{j}+\varepsilon_{n n n} \sum_{i=1}^{M} \rho_{i} \sum_{j n n n i} \rho_{j},
$$

where $\varepsilon_{n n}$ and $\varepsilon_{n n n}$ are the strengths of the interactions between nearest-neighbor and next-nearest-neighbor particles, respectively. The term $\sum_{j n n i} \rho_{j}$ denotes the sum of the densities in lattice sites $j$ that are the nearest neighbors to the site $i$. Similarly, $\sum_{j n n n i} \rho_{j}$ denotes the sum over the nextnearest neighbors (see Fig. 2). We now set $\varepsilon_{n n}=\varepsilon$ and $\varepsilon_{n n n}=\varepsilon / 4$. This ratio $\varepsilon_{n n} / \varepsilon_{n n n}$ is not the value it would have if the Lennard-Jones potential were exactly applied, but it is the optimum ratio to obtain circular drops when solved in two dimensions (see Sec. VII C). ${ }^{17-19}$ Our definition captures the essence of the Lennard-Jones potential: the repulsive core is modeled by the onsite repulsion (one particle per lattice site) and the pair interaction terms crudely model the attractive forces. However, it is worth noting that even though the interaction energy between two well-separated $\left(r \gg r_{0}\right)$ particles can be very small, the net contribution from all such long-range interactions may be significant and neglecting them may result in the theory failing to describe some interesting physics.

\section{B. External potential}

We assume that the interaction potential between a particle and the particles that form the wall of the container is of the Lennard-Jones form, which decays for large $r$ as $v(r) \sim-r^{-6}$. Summing the potential between a single fluid particle with all of the particles in the wall yields a net potential that decays as $V(z) \sim-z^{-3}$ for $z \rightarrow \infty$, where $z$ is the

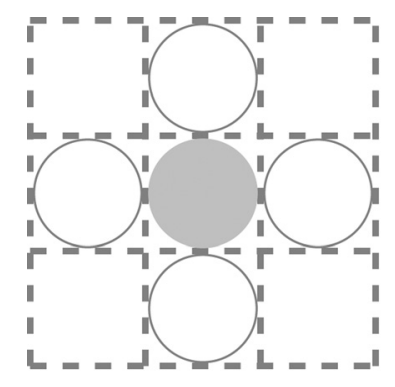

(a)

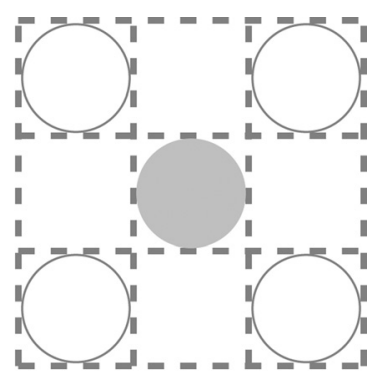

(b)
Fig. 2. The distinction between (a) the nearest neighbors (open circles) to a particle (grey circle) and (b) the next-nearest neighbors. 
perpendicular distance between the particle and the wall. We therefore assume that the wall exerts a potential of the form

$$
V_{i}= \begin{cases}\infty & \text { if } k<1 \\ -\varepsilon_{w} k^{-3} & \text { if } k \geq 1\end{cases}
$$

where $\varepsilon_{w}$ is the parameter that defines the attractive strength of the confining wall. The integer index $k$ is the distance, in units of lattice sites, of the particle from the wall.

\section{THE BULK FLUID PHASE DIAGRAM}

Before discussing the behavior of the fluid at this wall, we first calculate the phase diagram of the bulk fluid away from the influence of any interfaces. When the temperature $T$ is less than the critical temperature $T_{c}$, the fluid exhibits phase separation into a low-density gas and a high-density liquid. The binodal is the line in the phase diagram at which this transition occurs. Along the binodal, the liquid and the gas coexist in thermodynamic equilibrium, where the pressure, chemical potential, and temperature of the liquid and gas phases are equal.

The lattice gas model has a hole-particle symmetry that is not present in a continuum description, but that is useful for calculating the binodal. This symmetry arises from the fact that if we replace $n_{i}=1-h_{i}$, where $h_{i}$ is the occupation number for holes, then the form of Eq. (7) is unchanged. A hole is simply the absence of a particle: if $n_{i}=1$ then $h_{i}=0$. This symmetry leads to the densities of the coexisting gas and liquid, $\rho_{g}$ and $\rho_{l}$, respectively, to be related as

$$
\rho_{l}=1-\rho_{g} .
$$

We now follow the standard procedure for calculating the phase diagram, making use of the relation (41). From Eq. (38), the Helmholtz free energy per lattice site $f=F / M$, for a uniform fluid with density $\rho$, is

$$
f=k_{B} T[\rho \ln \rho+(1-\rho) \ln (1-\rho)]-\frac{5 \varepsilon}{2} \rho^{2},
$$

where $5 \varepsilon / 2=(1 / 2 M) \sum_{i, j} \varepsilon_{i j}=(1 / 2) \times 4\left(\varepsilon_{n n}+\varepsilon_{n n n}\right)$ is the sum of the particle interactions up to next-nearest neighbors. The pressure in the system is 5,21

$$
\begin{aligned}
P(\rho) & =-\left(\frac{\partial F}{\partial V}\right)_{T, N}=\rho \frac{\partial f}{\partial \rho}-f \\
& =-k_{B} T \ln (1-\rho)-\frac{5}{2} \varepsilon \rho^{2} .
\end{aligned}
$$

The binodal curve can be found by locating where the pressures of the two phases are equal. Invoking Eq. (41) and solving $P(\rho)=P(1-\rho)$ gives

$$
\frac{k_{B} T}{\varepsilon}=\frac{5(2 \rho-1)}{2[\ln \rho-\ln (1-\rho)]},
$$

which is plotted in Fig. 3(a). Note that the symmetry (41) greatly simplifies the calculation. This simple method is applicable only to the lattice fluid. More generally, one must solve for where the pressure and chemical potential are equal simultaneously. The maximum on the binodal corresponds to the critical point, above which there is no gas-liquid phase separation. From the symmetry (41), the density at the
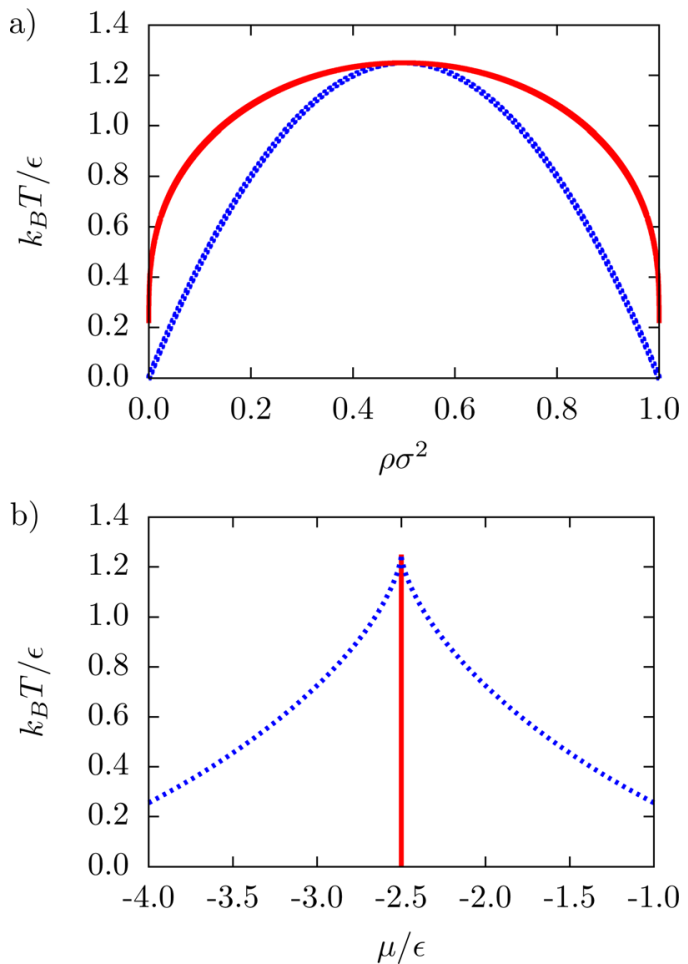

Fig. 3. The bulk fluid phase diagram for the $2 \mathrm{D}$ lattice fluid. The solid line is the binodal and the dashed line is the spinodal. In (a) we display the phase diagram in the dimensionless temperature $k_{B} T / \varepsilon$ versus density plane and in (b) we plot dimensionless temperature as a function of chemical potential.

critical point is $\rho=1 / 2$ and the critical temperature is found to be $T_{c}=5 \varepsilon / 4 k_{B}$.

The chemical potential can also be calculated from the Helmholtz free energy as 5,21

$$
\mu(\rho)=\left(\frac{\partial F}{\partial N}\right)_{T, V}=\frac{\partial f}{\partial \rho}=k_{B} T \ln \left(\frac{\rho}{1-\rho}\right)-5 \varepsilon \rho .
$$

On substituting Eq. (44) into Eq. (45), we find that the chemical potential at coexistence is

$$
\mu_{\mathrm{coex}}=-\frac{5}{2} \varepsilon
$$

which is plotted in Fig. 3(b). The spinodal is also plotted in Fig. 3. The spinodal denotes the locus in the phase diagram where the compressibility is zero-within this curve the fluid is unstable and spontaneous phase separation occurs. The spinodal is obtained from the condition

$$
\frac{\partial^{2} f}{\partial \rho^{2}}=0
$$

which, from Eq. (42), gives an expression for the density dependence of the temperature along the spinodal

$$
\frac{k_{B} T}{\varepsilon}=5 \rho(1-\rho),
$$

also plotted in Fig. 3(a). The spinodal can also be obtained as a function of $\mu$ from Eqs. (45) and (48); the result is shown in Fig. 3(b). 


\section{Exercise:}

Calculate the binodal for the case when there are only nearest-neighbor interactions. What is the critical temperature?

\section{AN ITERATIVE METHOD FOR CALCULATING THE DENSITY PROFILE}

We return now to the inhomogeneous fluid in the presence of an external potential. The equilibrium density profile is that which minimizes $\hat{\Omega}$ in Eq. (38); it is the set $\left\{\rho_{i}\right\}$ that satisfies, for all $i$,

$$
\frac{\partial \hat{\mathbf{\Omega}}}{\partial \rho_{i}}=0
$$

Performing this differentiation and rearranging gives the set of coupled equations

$$
\rho_{i}=\left(1-\rho_{i}\right) \exp \left[\beta\left(\mu+\varepsilon \sum_{j n n i} \rho_{j}+\frac{\varepsilon}{4} \sum_{j n n n i} \rho_{j}-V_{i}\right)\right],
$$

which can be solved iteratively for the profile $\left\{\rho_{i}\right\}$. An initial approximation is required and the closer this is to the true solution, the better. One option for the initial profile is $\rho_{i}=\exp \left[\beta\left(\mu-V_{i}\right)\right]$, the exact result in the lowdensity (ideal-gas) limit; otherwise, we can simply guess a likely profile. We can also use values from previous state points as an initial approximation when calculating at several state points successively, incrementing one parameter each time. With a suitable initial approximation for $\left\{\rho_{i}\right\}$, Eq. (50) can then be iterated until convergence is achieved.

It is often necessary during each iterative step to mix the result from evaluating the right-hand-side of Eq. (50), $\rho_{i}^{\text {rhs }}$, in a linear combination with the result from the previous iteration, $\rho_{i}^{\text {old }}$, so that

$$
\rho_{i}^{\text {new }}=\alpha \rho_{i}^{\text {rhs }}+(1-\alpha) \rho_{i}^{\text {old }},
$$

where $\alpha$ may be small, typically in the range $0.01<\alpha<0.1$. Such mixing has the effect that only small steps are taken towards the minimum with each iteration. Omitting this mixing (i.e., taking $\alpha=1$ ) can give a $\rho_{i}^{\text {new }}$ that falls outside of the range $(0,1)$, and once this happens the iterative routine breaks down.

\section{A. Normalizing the density profile}

To describe an enclosed (canonical) system with fixed $N$, we can treat Eq. (38) as a constrained minimization: minimizing the Helmholtz free energy

$$
\begin{aligned}
F= & k_{B} T \sum_{i=1}^{M}\left[\rho_{i} \ln \rho_{i}+\left(1-\rho_{i}\right) \ln \left(1-\rho_{i}\right)\right] \\
& -\frac{1}{2} \sum_{i, j} \varepsilon_{i j} \rho_{i} \rho_{j}+\sum_{i=1}^{M} V_{i} \rho_{i},
\end{aligned}
$$

subject to the constraint that

$$
N=\sum_{i=1}^{M} \rho_{i}
$$

The chemical potential $\mu$ is then the Lagrange multiplier associated with this constraint. To enforce this constraint when iteratively calculating the density profile $\left\{\rho_{i}\right\}$, we modify the method described above and at each iteration following the mixing step (51) the profile is renormalized $\rho_{i}^{\text {norm }}=A \rho_{i}^{\text {new }}$, with

$$
A=N\left(\sum_{i=1}^{M} \rho_{i}^{\text {new }}\right)^{-1}
$$

so that the constraint (53) is satisfied.

\section{B. Boundary conditions}

At the wall, the boundary conditions $(\mathrm{BC})$ for the density profile are straightforward: we simply set $\rho_{i}=0$ for all lattice sites $i$ "inside" the wall [for $k<1$ in Eq. (40)]. On the boundaries perpendicular to the wall we normally use periodic $\mathrm{BC}$, where it is assumed that the nearest neighbor of a lattice site on the boundary is the lattice site on the opposite boundary. For the boundary opposite the wall, periodic BC would create an artificial substrate (so that the fluid is confined in a capillary, between two walls). This does not cause a problem in sufficiently large systems; however, a more efficient solution is to assume that the fluid is uniform beyond the boundary opposite the wall with a specified density (e.g., that of the bulk gas).

\section{TYPICAL SOLUTIONS}

We now present results using the lattice gas model, which are typical of many DFT models for a fluid exhibiting gasliquid phase separation. After determining the equilibrium density profile using the iterative method described above, we can then calculate thermodynamic quantities such as the interfacial tension or the adsorption at the wall. The adsorption is defined as

$$
\Gamma=\sum_{i=1}^{M}\left(\rho_{i}-\rho_{b}\right)
$$

where $\rho_{b}$ is the bulk density, which is obtained by solving Eq. (45) for $\rho$. Note that for a 3D fluid $\Gamma$ is an excess number per area, whereas in 2D it is an excess per length. Also, the formula in Eq. (55) is true only when $\sigma=1$. By calculating results in the grand canonical ensemble we can track how the adsorption changes with $\mu$ (Sec. VII B). Working in the canonical ensemble, we can find drop profiles and calculate the contact angle that the liquid drop makes with a substrate ${ }^{22}$ (Sec. VII C). From these results, we can also determine if the liquid wets the substrate. We characterize a liquid as wetting a substrate when, at liquid-gas coexistence, a macroscopically thick layer of the liquid forms between the gas and the substrate. ${ }^{23-31}$ Grand canonically, this is characterized by $\Gamma \rightarrow \infty$ as coexistence is approached $\left(\mu \rightarrow \mu_{\text {coex }}^{-}\right)$. Treating the system canonically, the number of particles is fixed at $N=\sum_{i=1}^{M} \rho_{i}$ (using the normalization discussed in Sec. VI A) so that $\Gamma$ is fixed and we characterize wetting by the contact angle that a liquid drop makes with the substrate. In both 
cases, wetting occurs only when it is energetically beneficial, i.e., the liquid wetting the substrate is the state in which the system has the least energy.

\section{A. One-dimensional model}

So far, we have assumed for simplicity that the fluid is in two dimensions. However, since the density profile is defined as an average over all possible configurations [see Eq. (15)], if the external potential varies in only one direction [such as the potential in Eq. (40)], then so must the density profile. This is, of course, also the case for the three-dimensional fluid. The equilibrium density profile must have the same symmetry as the external potential and so we can reduce the DFT equations to be solved, Eq. (50), to a one-dimensional (1D) system consisting of a line of lattice sites extending perpendicularly away from the wall. We do this by summing over the interactions in the (transverse) direction in which the density does not vary, as illustrated in Fig. 4. This maps the 2D system onto an effective $1 \mathrm{D}$ system with renormalized interactions between lattice sites, and also introduces an effective on-site interaction. A similar mapping can also be done for the $3 \mathrm{D}$ fluid.

\section{Exercise:}

Implement the procedure described in Sec. VI on a computer to calculate the density profiles for this effective 1D model. (ii) Modify your computer code to solve for the density profile in $2 \mathrm{D}$. (iii) Compare the results from the $1 \mathrm{D}$ and $2 \mathrm{D}$ calculations. Are they the same?

\section{B. Adsorption at the wall}

In Fig. 5(a), we illustrate how the adsorption at the wall $\Gamma$ changes as the chemical potential is increased $\left(\mu \rightarrow \mu_{\text {coex }}^{-}\right)$to approach the coexistence value in Eq. (46) from below. When $\mu<\mu_{\text {coex }}$ the bulk phase (away from the wall) is the gas phase, but for a wall to which the particles are attracted the density at the substrate can be higher. As $\mu \rightarrow \mu_{\text {coex }}^{-}$the adsorption increases, either diverging $(\Gamma \rightarrow \infty)$ if the liquid wets the wall or remaining finite if the liquid does not wet the wall. As $T$ or $\varepsilon_{w}$ is changed, there is often a phase transition from one regime to the other, termed the "wetting transition.",23-31

The adsorption results in Fig. 5(a) are calculated for fixed $\beta \varepsilon=1.2$. When the strength of the attraction due to the wall

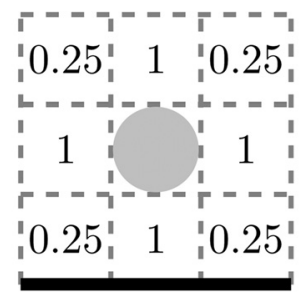

(a) Two Dimensional

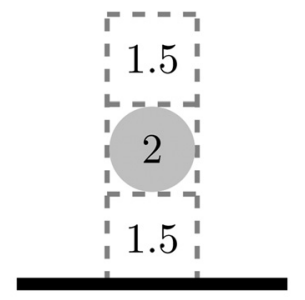

(b) One Dimensional
Fig. 4. Illustration of the mapping of the full 2D particle pair interactions onto an effective 1D system. The numbers represent the contribution towards the potential (in units of $\varepsilon$ ) from that particular lattice site with reference to the shaded particle in the center. The $2 \mathrm{D}$ case on the left is that discussed in Sec. IV A and on the right we display the resulting effective potential after mapping this system to $1 \mathrm{D}$.
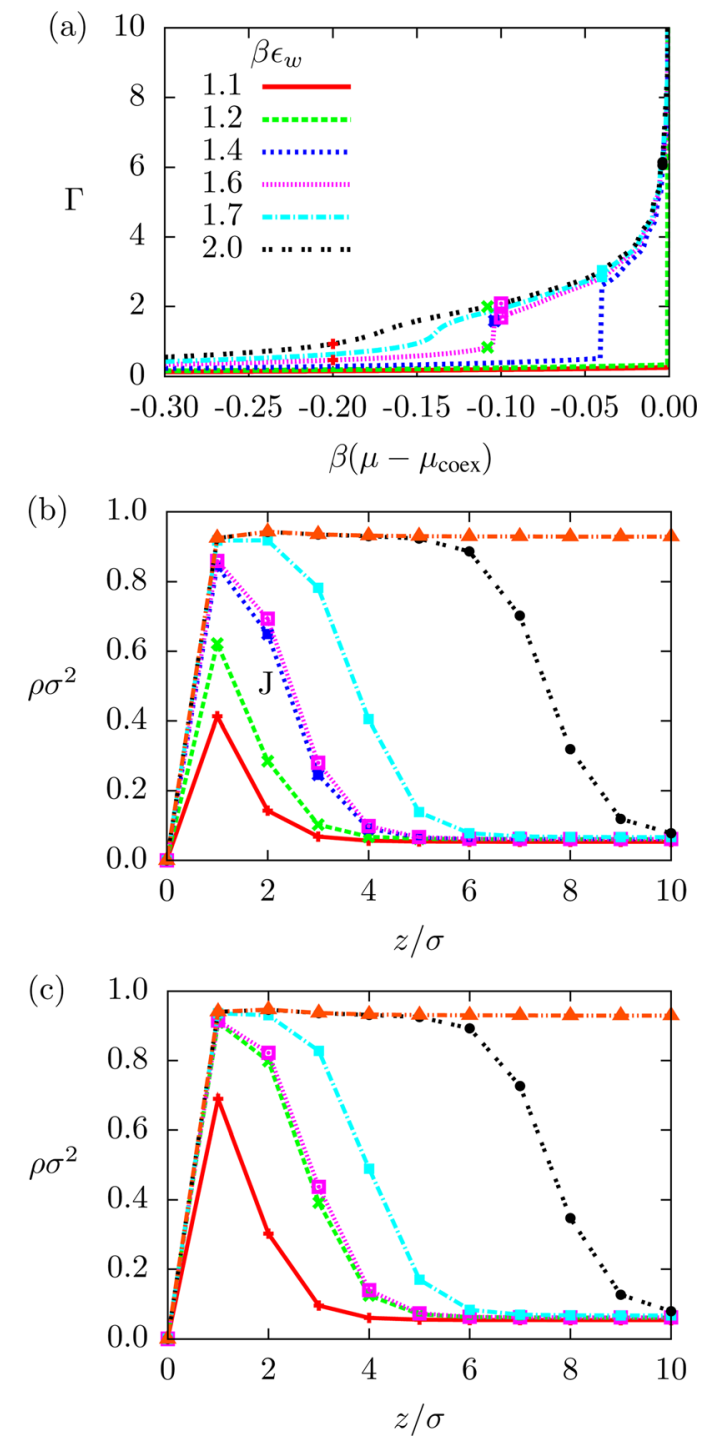

Fig. 5. (Color online) (a) The adsorption at the wall as the chemical potential $\mu \rightarrow \mu_{\text {coex }}^{-}$for various different values of the wall attraction strength parameter $\varepsilon_{w}$, as given in the key, for $\beta \varepsilon=1.2$. In (b) we display some of the corresponding density profiles for $\beta \varepsilon_{w}=1.6$, at $\beta\left(\mu-\mu_{\text {coex }}\right)=-0.2,-0.108$, $-0.104,-0.1,-0.04,-0.004$, and 0 . The "J" indicates where the profiles jump discontinuously as $\mu$ is varied. In (c) we display density profiles for $\beta \varepsilon_{w}=2$, at $\beta\left(\mu-\mu_{\text {coex }}\right)=-0.2,-0.1,-0.04,-0.004$, and 0 . In this case, the profiles vary continuously with $\mu$. The points in (a) denote states corresponding to the profiles in (b) and (c), with matching styles (online).

is weak $\left(\beta \varepsilon_{w}<1.2\right)$ the liquid does not wet the wall and the adsorption remains finite at coexistence $\left(\mu=\mu_{\text {coex }}\right)$. However, for stronger attraction $\left(\beta \varepsilon_{w}>1.2\right)$ the wetting film thickness diverges as $\mu \rightarrow \mu_{\text {coex }}^{-}$. To compute these results, a value of $\mu$ is set and the equilibrium profile $\left\{\rho_{i}\right\}$ is found. The value of $\mu$ is then incremented and the previous equilibrium solution used as the initial approximation for the next solution. At each state point, the adsorption is calculated via Eq. (55).

An interesting thing to note is that for some values of $\varepsilon_{w}$ the adsorption diverges continuously (see, e.g., the case for $\beta \varepsilon_{w}=2$ ), but for other values there is a discontinuous jump in $\Gamma$. This jump is a result of crossing the "pre-wetting line." 23-31 We see the beginnings of this jump as a continuous "shoulder" for $\beta \varepsilon_{w}=1.7$. As $\beta \varepsilon_{w}$ decreases the jump becomes larger and occurs closer to $\mu=\mu_{\text {coex }}$. The 
adsorption for $\beta \varepsilon_{w}=1.2$ remains very small until almost exactly at $\mu=\mu_{\text {coex }}$, where it jumps to a large value. We also observe some smaller discontinuous changes in $\Gamma$ occurring after the main pre-wetting jump. These smaller jumps are "layering transitions" and are due to an additional layer of particles being discontinuously added to the adsorbed liquid film. While layering transitions are observed in more sophisticated DFT theories, the underlying lattice in the present model leads to an unrealistic amplification of this effect. Figures 5(b) and 5(c) illustrate how the density profile changes as $\mu \rightarrow \mu_{\text {coex }}^{-}$for values of $\varepsilon_{w}$ that lead to wetting of the wall. We see a layer of the liquid phase appearing against the wall, increasing in thickness as coexistence is approached. In Fig. 5(b), we also see how the density profiles change discontinuously as the pre-wetting line is crossed.

Tracking the adsorption is useful for understanding how the fluid behaves as coexistence is approached. However, it ought not be used as the sole indicator of the wetting behavior. One should also calculate the grand potential $\Omega$. It can often arise that a given density profile actually corresponds only to a local minimum of $\Omega$, but in fact the global minimum corresponds to a different density profile (e.g., with higher adsorption).

\section{Exercise:}

Set $\beta \varepsilon_{w}=1.3$ and calculate the density profile at coexistence $\mu=\mu_{\text {coex }}$, for a range of different "temperatures" $\beta \varepsilon$. What do you find?

\section{Drop profiles and surface tensions}

We now return to the full 2D model and show typical density profiles corresponding to drops of liquid on a surface acting with the potential in Eq. (40). We treat the system canonically; that is, we normalize the system as discussed in Sec. VI A. We also break translational symmetry, placing the center-of-mass at the horizontal midpoint. ${ }^{32,33}$

The approximation for initiating our iterative procedure consists of setting the density $\rho_{i}=\rho_{g}$ everywhere, except in a region in the middle of the system next to the wall, where we set $\rho_{i}=\rho_{l}$. The final size of the liquid drop is determined by the average density value selected for the normalization step. The boundary conditions are as described in Sec. VI B, with $\rho_{i}=\rho_{g}$ along the top boundary (opposite to the wall).

In Fig. 6, we display some typical density profiles for various values of $\beta \varepsilon_{w}$, calculated on a $100 \times 40$ lattice for the fluid with temperature $\beta \varepsilon=1.2$. The adsorption (i.e., particle number) is the same in each panel. The liquid drop spreads out more on the substrate with larger values of $\varepsilon_{w}$. The contact angle $\theta$ that the drop makes with the substrate decreases as $\varepsilon_{w}$ is increased, so that the drop becomes broader until complete wetting occurs at $\beta \varepsilon_{w} \approx 1.2$, when the drop becomes a flat film.

The interfacial tension (or "surface tension" in 3D) is the excess free energy due to the presence of an interface between two phases. In the present system, there are three phases: the solid (wall), liquid, and gas. Thus, there are three different interfacial tensions for the wall-liquid, wall-gas, and liquid-gas interfaces, $\gamma_{w l}, \gamma_{w g}$, and $\gamma_{l g}$, respectively. For just the liquid and gas together, the interfacial tension $\gamma_{l g}$ leads to a liquid drop surrounded by the gas to form a circle (in $2 \mathrm{D}$, or a sphere in $3 \mathrm{D}$ ), because this shape minimizes the interfacial area and therefore minimizes its contribution to the free energy. When the wall is present, which cannot

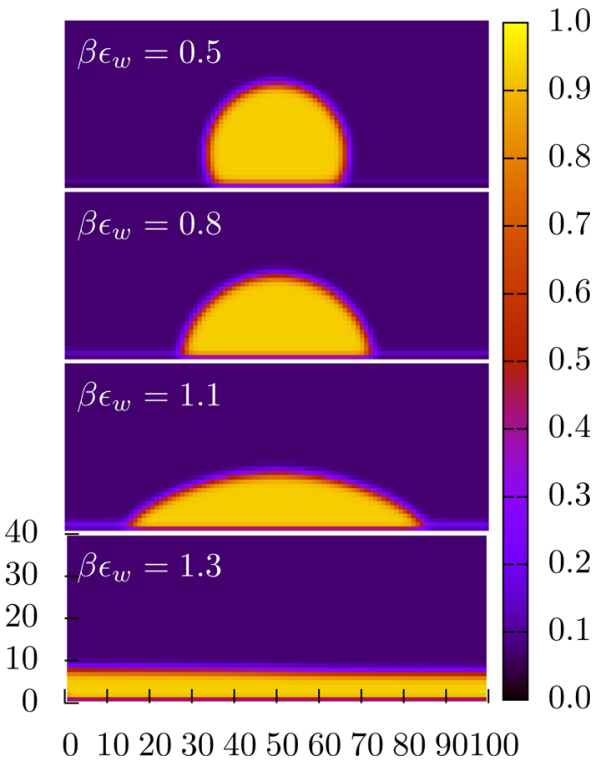

Fig. 6. Drop density profiles for fixed $\beta \varepsilon=1.2$ with varying values of $\beta \varepsilon_{w}$. The drops spread out with increasing $\beta \varepsilon_{w}$ until a flat film forms beyond the wetting transition.

change, the gas and liquid must arrange themselves so as to minimize the free energy. The resulting configuration depends on the values of the interfacial tensions. The equilibrium value of the contact angle is given by Young's Equation $^{23-28}$

$$
\gamma_{l g} \cos \theta=\gamma_{w g}-\gamma_{w l}
$$

which can be understood by considering the balance of forces due to the interfacial tensions at the point where the three phases meet (this is the contact line in 3D).

Within the present microscopic theory, we can calculate the interfacial tensions, enabling a comparison with the macroscopic arguments that lead to Eq. (56). To determine $\gamma_{l g}$, calculate the density profile through the interface between a semi-infinite slab of the liquid and a semi-infinite slab of the gas. This is obtained in the same manner as the density profiles at the wall in Sec. VII B, but in this case we remove the wall (setting $V_{i}=0$ for all $i$ ) and set the boundary condition to $\rho_{i}=\rho_{l}$; at the other end, $\rho_{i}=\rho_{g}$ as before. The initial guess for the density profile consists of setting $\rho_{i}=\rho_{l}$ in one half of the system and $\rho_{i}=\rho_{g}$ in the other half and, of course, we must set $\mu=\mu_{\text {coex }}$. From the resulting profile $\left\{\rho_{i}\right\}$, we then calculate the grand potential $\Omega$ from Eq. (38). The grand potential without the interface (either full of gas or full of just the liquid) is

$$
\Omega_{0}=-p V
$$

where $p$ is the pressure and $V$ is the volume (system size). The interfacial tension is then

$$
\gamma_{l g}=\frac{\Omega-\Omega_{0}}{A},
$$

where $A$ is the length of the 2D interface. The wall-gas and wall-liquid interfacial tensions are calculated in a similar manner except we retain the wall potential and we initialize the system entirely with either the gas or the liquid density, respectively. Note that above we have solely discussed the 
Table I. Interfacial tensions and contact angle $\theta$ from Eq. (56), for different values of the wall attraction strength $\varepsilon_{w}$.

\begin{tabular}{lrrr}
\hline \hline$\beta \varepsilon_{w}$ & $\sigma \beta \gamma_{w l}$ & $\sigma \beta \gamma_{w g}$ & $\theta$ \\
\hline 0.5 & 0.12 & -0.05 & $115^{\circ}$ \\
0.8 & -0.16 & -0.09 & 79 \\
1.0 & -0.36 & -0.13 & $52^{\circ}$ \\
1.3 & -0.68 & -0.23 & 0 \\
\hline \hline
\end{tabular}

interfacial tensions for a straight interface. For curved interfaces, the tensions depend on the curvature and the calculations become more involved. A discussion of some of the key issues can be found in Ref. 34 and references therein.

When $\beta \varepsilon=1.2$, the gas-liquid interfacial tension $\gamma_{l g}$ is $0.38 k_{B} T / \sigma$, corresponding to the case for the profiles displayed in Fig. 6. The other interfacial tensions are given in Table I, together with the resulting contact angle from Eq. (56). These values are in good agreement with the contact angle one can observe from the density profiles in Fig. 6. However, these profiles have a diffuse interface so there is always some uncertainty in the location of the contact line. As $\varepsilon_{w}$ is increased the drop spreads because it is energetically beneficial to do so. Complete spreading (wetting) occurs only when the sum $\gamma_{l g}+\gamma_{w l}$ is less than $\gamma_{w g}$.

\section{Exercise:}

Calculate one of the density profiles from Fig. 6, implementing the normalization procedure introduced in Sec. VI A and then plot the density contour $\rho=\left(\rho_{g}+\rho_{l}\right) / 2$, which corresponds to the mid-point of the liquid-gas interface. Where on this curve does the contact angle agree with the macroscopic result in Eq. (56)? Is it where you would expect?

\section{CONCLUSIONS}

We have presented a derivation of a simple lattice gas model DFT and discussed typical applications. Working with this model gives a good hands-on introduction to many of the important ideas behind DFT and gives a platform to learn about different aspects of inhomogeneous fluids such as phase diagrams, adsorption, wetting, and surface tensions. Studying this "toy-model" gives students good insight and a feeling for the physics of inhomogeneous liquids, leaving them in a good position to continue on and study the "real thing."2-6,8-12

\section{ACKNOWLEDGMENTS}

A.P.H. acknowledges support through a Loughborough University Graduate School Studentship. A.J.A. thanks all the students who have done projects with him modeling inhomogeneous liquids with this lattice-gas DFT or variants of it. This paper is largely based on informal lectures and many discussions with Mark Amos, Blesson Chacko, Chris Chalmers, William Dewey, Mark Robbins, and Sen Tian.

${ }^{1}$ P.-G. de Gennes, F. Brochard-Wyart, and D. Queré, Capillarity and Wetting Phenomena: Drops, Bubbles, Pearls, Waves (Springer, New York, 2004).

${ }^{2}$ Fundamentals of Inhomogeneous Fluids, edited by D. Henderson (Marcel Dekker, New York, 1992).
${ }^{3}$ J. S. Rowlinson and B. Widom, Molecular Theory of Capillarity (Dover, New York, 2002).

${ }^{4} \mathrm{H}$. Ted Davis, Statistical Mechanics of Phases, Interfaces, and Thin Films (Wiley-VCH, New York, 1996).

${ }^{5}$ J.-P. Hansen and I. R. McDonald, Theory of Simple Liquids, 4th ed. (Elsevier, Amsterdam, 2013).

${ }^{6} \mathrm{R}$. Evans, "The nature of the liquid-vapour interface and other topics in the statistical mechanics of non-uniform, classical fluids," Adv. Phys. 28(2), 143-200 (1979).

${ }^{7}$ R. Evans, "Density Functionals in the Theory of Nonuniform Fluids," in Fundamentals of Inhomogeneous Fluids, edited by D. Henderson (Marcel Dekker, New York, 1992), pp. 85-176.

${ }^{8}$ J. F. Lutsko, "Recent developments in classical density functional theory," Adv. Chem. Phys. 144, 1-92 (2010).

${ }^{9}$ J. Wu and Z. Li, "Density-functional theory for complex fluids," Annu. Rev. Phys. Chem. 58, 85-112 (2007).

${ }^{10} \mathrm{~J}$. Wu, "Density functional theory for chemical engineering: From capillarity to soft materials," AIChE J. 52(3), 1169-1193 (2006).

${ }^{11}$ P. Tarazona, J. A. Cuesta, and Y. Martínez-Ratón, "Density functional theories of hard particle systems," Lect. Notes Phys. 753, 247-341 (2008).

${ }^{12}$ H. Löwen, "Density Functional Theory for Inhomogeneous Fluids II (Freezing, Dynamics, Liquid Crystals)," Lecture Notes, 3rd Warsaw School of Statistical Physics (Warsaw U.P., Warsaw, 2010), pp. 87-121.

${ }^{13}$ The students involved in these projects have generally been in the final year of either a three-year bachelors degree or a four-year masters degree, in either Mathematics \& Physics or straight Mathematics. However, twice these activities were given as summer projects to very good students at an earlier stage in their studies, which worked well too. The final-year projects are typically supposed to be around 200 or $300 \mathrm{~h}$ work over the academic year, including meeting with the supervisor for roughly one hour per week. In order to get students started and introduce to them the relevant mathematics and physics for these projects, $2-3 \mathrm{~h}$ of informal introductory lectures are given; Secs. I-V of this paper are based on these lectures. Some students are also given a computer code written in Maple that implements the method described in Sec. VI, for the fluid in 1D with just nearest neighbor interactions, which the student then modifies to tackle their particular problem.

${ }^{14} \mathrm{M}$. Plischke and B. Bergersen, Equilibrium Statistical Mechanics, 3rd ed. (World Scientific, Singapore, 2006).

${ }^{15}$ L. E. Reichl, A Modern Course in Statistical Physics, 3rd ed. (Wiley$\mathrm{VCH}$, Weinheim, 2009).

${ }^{16}$ M. Schoen and S. Klapp, "Nanoconfined fluids: Soft matter between two and three dimensions," in Reviews in Computation Chemistry, edited by K. B. Lipkowitz and T. Cundari (Wiley, New York, 2007), Vol. 24.

${ }^{17}$ M. J. Robbins, "Describing colloidal soft matter systems with microscopic continuum models," Ph.D. thesis, Loughborough University, 2012, $<$ https://dspace.lboro.ac.uk/2134/9383>.

${ }^{18}$ M. J. Robbins, A. J. Archer, and U. Thiele, "Modelling the evaporation of thin films of colloidal suspensions using Dynamical Density Functional Theory," J. Phys. Condens. Matter 23, 415102-1-18 (2011).

${ }^{19}$ S. Fomel and J. F. Claerbout, "Exploring three-dimensional implicit wavefield extrapolation with the helix transform," SEP Rep. 95, 43-60 (1997); available at http://www.reproducibility.org/RSF/book/sep/findif/paper.pdf.

${ }^{20} \mathrm{D}$. Chandler, Introduction to Modern Statistical Mechanics (Oxford U.P., New York, 1987).

${ }^{21}$ F. Mandl, Statistical Physics, 2nd ed. (John Wiley \& Sons, Padstow, 1988).

${ }^{22}$ In the grand canonical ensemble a liquid drop is not an equilibrium statedrops either evaporate or grow, depending on the value of $\mu$.

${ }^{23}$ R. Evans, "Micoroscopic theories of simple fluids and their interfaces," in Liquids at Interfaces, Les Houches Session XLVIII, 1988, edited by J. Charvolin, J. F. Joanny, and J. Zinn-Justin (Elsevier, Amsterdam, 1990), pp. 1-98.

${ }^{24}$ M. Schick, "Introduction to Wetting Phenomena," in Liquids at Interfaces, Les Houches Session XLVIII, 1988, edited by J. Charvolin, J. F. Joanny, and J. Zinn-Justin (Elsevier, Amsterdam, 1990), pp. 415-497.

${ }^{25} \mathrm{~S}$. Dietrich, "Wetting phenomena," in Phase Transition and Critical Phenomena, edited by C. Domb and J. L. Lebowitz (Academic Press, London, 1988), Vol. 12, pp. 2-218.

${ }^{26}$ D. Bonn and D. Ross, "Wetting transitions," Rep. Prog. Phys. 64, 1085-1163 (2001).

${ }^{27}$ D. Bonn, J. Eggers, J. Indekeu, J. Meunier, and E. Rolley, "Wetting and spreading," Rev. Mod. Phys. 81, 739-805 (2009).

${ }^{28}$ V. M. Starov and M. G. Velarde, "Surface forces and wetting phenomena," J. Phys. Condens. Matter 21, 464121-1-11 (2009). 
${ }^{29}$ A. O. Parry, "Three-dimensional wetting revisited," J. Phys. Condens. Matter 8, 10761-10778 (1996).

${ }^{30}$ R. Pandit, M. Schick, and M. Wortis, "Systematics of multilayer adsorption phenomena on attractive substrates," Phys. Rev. B 26, 5112-5140 (1982).

${ }^{31}$ E. Bruno, U. M. B. Marconi, and R. Evans, "Phase transitions in a confined lattice gas: Prewetting and capillary condensation," Physica A 141, 187-210 (1987).
${ }^{32}$ D. Reguera and H. Reiss, "The role of fluctuations in both density functional and field theory of nanosystems," J. Chem. Phys. 120, 2558-2264 (2004).

${ }^{33}$ A. J. Archer and A. Malijevsky, "On the interplay between sedimentation and phase separation phenomena in two-dimensional colloidal fluids," Mol. Phys. 109, 1087-1099 (2011).

${ }^{34}$ M. C. Stewart and R. Evans, "Wetting and drying at a curved substrate: Long-ranged forces,” Phys. Rev. E 71, 011602-1-14 (2005).

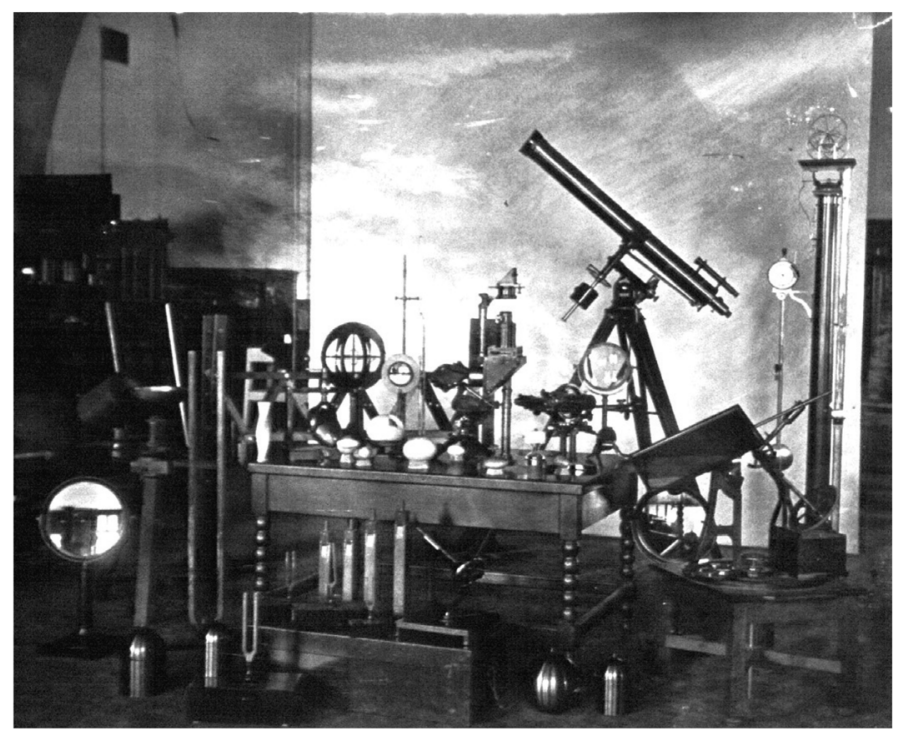

\section{Apparatus Collection at West Point}

In this picture, taken ca. 1900, see if you can find: Tuning forks on resonators, Savart's resonance apparatus, Telescope, Overhead projector, Heliostat, Helmholtz resonators, Saccharimeter, Organ pipes, Giant tuning fork. (Notes by Thomas B. Greenslade, Jr., Kenyon College) 